\title{
Cognitive orientation in substance use disorders and chronic cigarette smoking
}

\author{
Daniel Stein', Anca Ram ${ }^{1,2}$, Sivan Sofer ${ }^{3}$ and Shulamith Kreitler ${ }^{1,4 *}$ \\ ${ }^{1}$ Sackler Faculty of Medicine, University of Tel Aviv, The Chaim Sheba Medical Center, Tel Hashomer, Israel \\ ${ }^{2}$ Sackler Faculty of Medicine, University of Tel Aviv, Ness Ziona Mental Health Center, Ness Ziona, Israel \\ ${ }^{3}$ Department of Psychology, University of Haifa, Haifa, Israel \\ ${ }^{4}$ School of Psychological Sciences, Tel-Aviv University, Tel Aviv, and Psychooncology Research Center, Sheba Medical Center, Tel-Hashomer, Israel
}

\begin{abstract}
We sought to identify cognitive-motivational dispositions for addictiveness in females with different addictions. For this purpose we assessed 20 women with substance use disorders (SUDs), 19 women who were routinely smoking at least 10 cigarettes daily for several years, and 24 control women. Cognitive-motivational dispositions were assessed with the 108-item Cognitive Orientation of Addictive Tendencies Questionnaire (COAT-Q), that converges to 25 content-related motivational themes and four basic belief types. Anxiety, depression, and addictiveness were also assessed. We found that compared with the controls, the SUD patients scored higher on addictiveness, depression, anxiety, all four COAT-Q-beliefs types, and three COAT-Q-theme clusters: dissociation from the self and from contacts with others, rejecting bad parts of the self, and the coexistent wish and fear of being loved. The chronic smokers scored mostly in-between the SUD patients and controls. These findings suggest that specific cognitive motivational dispositions may orient towards addictive behaviours in SUDs and chronic smoking.
\end{abstract}

\section{Introduction}

Substance use is among the most critical issues facing society today [1]. The reasons for use and abuse of drugs likely involve complex interactions among physiologic/genetic, psychological, and environmental factors [1]. Personality tendencies may fulfill a central role in the predisposition to substance misuse [2]. Although the findings with respect to personality attributes in substance use disorders (SUDs) are still inconclusive, elevations on the Eysenck Addiction Scale [3], and on the Minnesota Multiphasic Personality Inventory (MMPI) scales of social withdrawal, anger, impulsivity, depression and anxiety have been found to differentiate SUD patients from individuals with other psychopathologies and/or from normal controls [4,5].

In addition, elevated rates of DSM-IV [6] Cluster B personality disorders, particularly antisocial and borderline personality disorders, are found in SUD individuals in comparison to healthy controls [7]. Several personality attributes of seemingly two opposite categories have also been identified across a broad range of addictions. One category includes deficient regulation and elevated stress reactivity, sensation seeking, novelty seeking, depression, anxiety, anger and impulsivity, and the other includes increased harm avoidance anhedonia, dependency, alexithymia, and low self-esteem [4,7-10].

Most of the research on the role of personality attributes in addiction is concerned with behavioral and emotional disturbances. Still, there is evidence that focusing on the cognitive-motivational dispositions underlying addiction-related behaviors may increase our understanding about the dynamics underlying the personality attributes associated with addiction-related behaviors. The cognitiveorientation (CO) theory $[11,12]$ may provide a theoretical and empirical framework for this purpose, as it has the potential to identify unconscious motivational dispositions that shape specific behavioral intents, which can, in turn, evoke specific behaviors. Indeed, studies assessing $\mathrm{CO}$ motivations underlying behavioral manifestations in diverse medical and psychiatric morbidities (e.g., schizophrenia, and eating disorders) $[13,14]$ have found high specificity and sensitivity, namely these motivations show considerable variability in different behaviors, but are replicable in different examinations of the same behavior.

The specificity of the $\mathrm{CO}$ model in orienting towards different behaviors is based on the manner in which the assessment tool, the Cognitive Orientation Questionnaire (COQ) is constructed [11,15]. In the first stage, individuals with a specific disorder and controls are interviewed with a standardized interview that identifies the general interpersonal and specific personal meanings of behaviors that are of relevance in this disorder. Those responses appearing in the interviews of at least $50 \%$ of the interviewees with the disorder and in less than $10 \%$ of those without it are included as statements in the COQ. From the viewpoint of form, these statements are phrased according to four belief types:

1. Beliefs about the self (COQ-BS) - does the individual see himself/ herself as capable to execute a specific behavior.

2. Beliefs about norms and rules (COQ-N) - is the specific behavior acceptable and allowed.

Correspondence to: Shulamith Kreitler, Head, Psychooncology Center, Sheba Medical Center, Tel Hashomer, Israel; Tel. +972-3-5304723; Fax: +972-35303031; E-mail: shulamith.kreitler@sheba.health.gov.il

Key words: addiction, substance use disorders, smoking, cognitive orientation

Received: March 28, 2017; Accepted: April 24, 2017; Published: April 27, 2017 
3. General beliefs (COQ-GB) - does the reality setting enable/ encourage the execution of the specific behavior. 4. Beliefs about goals (COQ-G) - does the individual want the specific behavior to happen. From the viewpoint of contents, the COQ-statements converge to different theme contents. The motivational dispositions directing towards a specific behavior consist of cells formed by the intersection of the four belief-types and specific theme-contents.

In the second phase, the questionnaire is tested for reliability and validity in another sample of the behavior studied. Validity is confirmed if the COQ differentiates individuals with the behavior in question from controls without it.

The present study is the first to assess the CO motivations underlying substance misuse. For this purpose we have used a modified version of the COQ of eating disorders (EDs) [14]. We have chosen to do so because studies in other domains have shown that $\mathrm{CO}$ questionnaires constructed for a specific behavior can be applied successfully for predicting other behaviors which share with the original behaviors at least a part of the underlying dynamics [16]. Along these lines of reasoning, ED patients have been found to show elevated addictiveness and to resemble SUD patients in major personality traits $[4,5,17]$. We have therefore used the CO-ED in SUD and ED patients [18], showing that ED patients resemble SUD patients and that both groups are markedly different from non-SUD non-ED controls in many motivational dispositions of the questionnaire. These findings have provided the theoretical background for preparing a modified COED-Q for addictive populations (see Method).

In the present study we assessed the cognitive motivations of individuals addicted to opoiods against a group of chronic cigarette smokers, and non-SUD non-smoking controls. We specifically aimed to study cigarette smokers because despite their overall normal functioning and adjustment [19], a profound physical and psychological addiction can develop in daily tobacco users $[1,20]$. We hypothesized that addictiveness-oriented motivations will be highest in SUD individuals, lowest in healthy non-SUD non-smoking controls and in a medium position in chronic cigarette smokers.

\section{Materials and methods}

\section{Patients}

The following female groups were included in the study:

1. Twenty patients diagnosed with DSM-IV [6] SUDs, treated at specific inpatient substance use centers in Israel. All were diagnosed as addicted to narcotic opiates, with $60 \%$ also abusing at least one other psychoactive substance (cannabis, amphetamines, cocaine, MDMA, LSD, or phencyclidine). At the time of evaluation, SUD patients were not engaged in active substance use for 2-6 consecutive months, as assessed by daily monitoring and weekly urine tests. SUD patients were excluded from the study if they ever had a bipolar disorder, schizophrenic spectrum disorder, organic brain disorder, ED, or any significant lifetime or current medical disorder. The age of the SUD patients at onset of the substance use was $21.37 \pm 6.1$ years respectively, and the duration of SUD $11.80 \pm 7.4$ years. Three of the 20 SUD patients were diagnosed additionally with a DSM-IV depressive disorder, 4 with an anxiety disorder, and another 3 with both a depressive and an anxiety disorder. No differences were found in any of the study's measures between SUD patients with and without a comorbid diagnosis.

2. Nineteen women who were routinely smoking at least 10 cigarettes daily (including at the time of evaluation) for a period of
$7.21 \pm 4.8$ years. These participants were required to have no lifetime or current psychiatric or medical disorders, including addiction/abuse to non-nicotine SUDs. Their age at onset of smoking was $16.79 \pm 3.5$ years, respectively.

3. Twenty-four non-smoking control females with no lifetime or current psychiatric or medical disorder, and no lifetime or current use of medications.

All participants and their parents, in the case of minors, signed a written informed consent after being explained about the goals and methodology of the study. The study was approved by the human subjects review committee of the Chaim Sheba Medical Center, Tel Hashomer, Israel.

\section{Interviews}

Demographic and clinical variables were assessed with a structured interview. DSM-IV (1994) SUDs and other Axis I disorders were diagnosed with the Structured Clinical Interview for DSM-IV Axis I Disorders-Patient Edition (SCID-I/P, Version 2.0) [21]

\section{Self-rating questionnaires}

Addictiveness was assessed with the 32-item Addiction Scale of the Eysenck Personality Questionnaire-Revised (EPQ-R-AS), found to successfully discriminate SUD individuals from healthy controls [3]. In the present study, the internal consistency of the EPQ-R-AS was $\alpha=.79$.

Depression and anxiety (state and trait) were assessed with the Depression and Anxiety Scales of the revised State-Trait Personality Inventory (STPI) [22].In the present study, the internal consistencies of the STPI were: $\alpha=.90$, and .92, for STPI-State Anxiety and Depression, respectively, and $\alpha=.88$, and .94 for STPI-Trait Anxiety, and Depression, respectively.

CO Motivational dispositions were assessed with the Cognitive Orientation of Addictive Tendencies Questionnaire (COAT-Q), constructed from the COQ of EDs [14]. For this purpose, we included only those themes from the CO-ED-Q questionnaire endorsed by at least $50 \%$ of the SUD patients and by less than $10 \%$ of the controls in a pretest sample of 20 SUD and 20 control participants. Thirtythree of the 48 themes of the CO-ED-Q (68.7\%) passed this criterion. In the second stage, we assessed these 33 COAT-Q themes in our SUD patients, cigarette smokers, and controls. Although a significant between-group difference was found for all 33 themes, only 25 passed the Bonferroni criterion for multiple comparisons $(p<.001$ for the significance level of $\mathrm{p}<.05$ in the case of 33 comparisons).

Accordingly, the final version of the COAT-Q includes 25 themes, and a total of 108 items for the four belief types: Beliefs about the self (COAT-Q-BS), about rules and norms (COAT-Q-N), general beliefs (COAT-Q-GB), and beliefs about goals (COAT-Q-G). For all COAT-Q items, elevated scores signify a greater tendency for addictiveness. In the present study, the internal consistencies of the COAT-Q are $\alpha=.86$, $.75, .78$, and .80 for the COAT-Q-BS, -N, -GB, and -G, respectively, and .81 for the combined COAT-Q.

\section{Procedure}

Participants were independently interviewed with the SCID$\mathrm{I} / \mathrm{P}$, Version 2.0 by two experienced master's level psychologists or psychiatrists. Final diagnoses were achieved after two psychiatric experts of the research team reviewed independently all interviews. Only those participants for whom there was a unanimous agreement as to their diagnosis entered the study. The COAT-Q, EPQ-R-AS, and 
STPI were administered individually to each participant by a different team of master's level psychologists.

\section{Statistical analysis}

Differences between SUD patients, cigarette smokers, and controls were analyzed with univariate analysis of variance (ANOVA) and Duncan post-hoc comparisons. Analysis of covariance (ANCOVA) was introduced to control for the influence of the between-group differences in age, education, anxiety, and depression on the differences in cognitive orientation. A Bonferroni's correction for multiple comparisons was set at $\mathrm{p}<.001$.

\section{Results}

Compared with the cigarette smokers and controls, the SUD patients were significantly older [31.15 \pm 7.7 years vs. $24.00 \pm 5.2$ years and $23.08 \pm 7.4$ years respectively, $F(2,63)=13.44, p<.0001]$ and had less years of schooling $[10.45 \pm 2.0$ years vs. $12.71 \pm 1.1$ years and $13.58 \pm$ 1.9 years, respectively, $\mathrm{F}(2,63)=18.74, \mathrm{p}<.0001]$.

Table 1 summarizes the findings for the STPI, EPQ-R-AS, and COAT-Q. .STPI-state anxiety and depression were elevated in the two research groups compared with the controls, whereas for the STPI-trait variables and addiction, the cigarette smokers were more disturbed than the controls, and the SUD patients more disturbed than the cigarette smokers. All STPI and EPQ-R-AS findings retained their significance following Bonferroni's correction, as well as when controlling for age and education (results not shown).

For the COAT-Q-BS and COAT-Q-N dimensions, the cigarette smokers scored higher than the controls, and the SUD patients scored higher than the cigarette smokers. For COAT-Q-GB, the two research groups scored higher than the controls, and for COAT-Q-G, the SUD patients scored higher than the two other groups.

A hierachical cluster analysis, including those 25 CAT-OQ-themes that yielded significant univariate between-group differences at the .001 level, identified three clusters.

Table 1. STPI-dimensions, addiction (EPQ-R-AS), and COAT-Q beliefs and theme-factors in SUD, cigarette smoking, and control female participants

\begin{tabular}{|c|c|c|c|c|}
\hline & $\begin{array}{c}\text { SUD patients } \\
(\mathrm{n}=23)\end{array}$ & $\begin{array}{c}\text { Cigarette } \\
\text { smokers } \\
(\mathrm{n}=19)\end{array}$ & $\begin{array}{c}\text { Controls } \\
(\mathrm{n}=24)\end{array}$ & $\mathrm{F}(2,65)$ \\
\hline Age & $31.15 \pm 7.7^{\mathrm{a}}$ & $24.00 \pm 5.2^{\mathrm{b}}$ & $23.08 \pm 7.4^{\mathrm{b}}$ & $13.44^{* * *}$ \\
\hline Education (years) & $10.45 \pm 2.0^{\mathrm{a}}$ & $12.71 \pm 1.1^{\mathrm{b}}$ & $13.58 \pm 1.9^{\mathrm{b}}$ & $18.74^{* * *}$ \\
\hline STPI-State Anxiety & $24.05 \pm 6.0^{\mathrm{a}}$ & $23.58 \pm 8 .^{2} \mathrm{a}$ & $17.67 \pm 5.0^{\mathrm{b}}$ & $7.94^{* *}$ \\
\hline STPI-State Depression & $21.90 \pm 5.8^{\mathrm{a}}$ & $21.84 \pm 8.4^{\mathrm{a}}$ & $13.87 \pm 3.4^{\mathrm{b}}$ & $13.12^{* * *}$ \\
\hline STPI-Trait Anxiety & $26.70 \pm 5.4^{\mathrm{a}}$ & $21.53 \pm 6.5^{\mathrm{b}}$ & $17.04 \pm 3.3^{\mathrm{c}}$ & $19.33^{* * *}$ \\
\hline STPI-Trait Depression & $25.70 \pm 5.9^{\mathrm{a}}$ & $21.21 \pm 8.2^{\mathrm{b}}$ & $17.12 \pm 3.3^{\mathrm{c}}$ & $11.28^{* * *}$ \\
\hline EPQ-R-AS & $52.70 \pm 4.3^{\mathrm{a}}$ & $47.22 \pm 4.8^{\mathrm{b}}$ & $40.83 \pm 3.6^{\mathrm{c}}$ & $44.25^{* * *}$ \\
\hline COAT-Q-BS & $155.75 \pm 18.2^{\mathrm{a}}$ & $128.00 \pm 24.4^{\mathrm{b}}$ & $111.12 \pm 11.2^{\mathrm{c}}$ & $32.94^{* * *}$ \\
\hline COAT-Q-N & $66.25 \pm 5.9^{\mathrm{a}}$ & $57.21 \pm 3.9^{\mathrm{b}}$ & $53.12 \pm 5.3^{\mathrm{c}}$ & $36.31^{* * *}$ \\
\hline COAT-Q-GB & $129.65 \pm 15.8^{\mathrm{a}}$ & $120.32 \pm 20.4^{\mathrm{a}}$ & $109.42 \pm 10.0^{\mathrm{b}}$ & $9.29^{* * *}$ \\
\hline COAT-Q-G & $63.70 \pm 5.4^{\mathrm{a}}$ & $53.00 \pm 5.4^{\mathrm{b}}$ & $51.37 \pm 3.6^{\mathrm{b}}$ & $40.07^{* * *}$ \\
\hline COAT-Q Cluster 1 & $131.95 \pm 15.0^{\mathrm{a}}$ & $113.00 \pm 18.5^{\mathrm{b}}$ & $100.25 \pm 11.4^{\mathrm{c}}$ & $24.56^{* * *}$ \\
\hline COAT-Q-Cluster 2 & $38.40 \pm 6.1^{\mathrm{a}}$ & $31.11 \pm 5.0^{\mathrm{b}}$ & $26.54 \pm 2.7^{\mathrm{c}}$ & $35.22^{* * *}$ \\
\hline COAT-Q-Cluster 3 & $40.55 \pm 4.8^{\mathrm{a}}$ & $34.79 \pm 5.0^{\mathrm{b}}$ & $29.54 \pm 3.6^{\mathrm{c}}$ & $33.52^{* * *}$ \\
\hline
\end{tabular}

Note: SUD-substance use disorder; STPI-State Trait Personality Interview; EPQ-R-ASEysenck Personality Questionnaire-Revised-Addiction Subscale; COAT-Q- Cognitive Orientation for Addictive Tendencies Questionnaire: BS-beliefs about self, N-beliefs about norms, GB-general beliefs, G-beliefs about goals;

$* *$-p $<.001 ; * * *$-p $<.0001$

Superscripts indicate the findings according to Duncan post hoc test. Scores with different superscripts differ significantly from each other in that row at $\mathrm{p} \leq .05$.
Cluster 1 includes 15 themes related to vulnerability, inclination towards extremity, and lack of control over ones life, as well as to the wish to commit oneself towards self-shaping and changing, perhaps because of lack of clarity about one's identity. It also includes themes related to fear of expressing one's opinions, a wish to avoid and overcome negative emotions, as well as not to gratify one's needs, to dissociate from one's body, to gain a sense of self-control, and to avoid close contacts, despite having difficulty to be alone. According to this cluster, the vulnerable, confused and out of control individual, feels that in order for a desirable personal change to happen, she has to dissociate herself from extreme, fearful, negative, and physicallyinvested personal emotions and needs, and from close contacts with others.

Cluster 2 includes 8 themes, relating to feeling evil, angry, guilty, impulsive, and inferior and to needs related to immediate gratification, self-punishment, and lack of enjoyment. We suggest this cluster to deal mainly with the rejection of the conceived evil self.

Cluster 3 includes 2 themes relating to wishing to be not recognized by others and to unconditioned love, thus dealing mainly with the coexistent wish and fear of being loved.

The findings for all four COQ-beliefs and three theme-clusters retained their significance level after controlling for age, education and STPI variables (results not shown).

A stepwise discriminant analysis with the COAT-Q-beliefs as predictors classified correctly $71.4 \%$ of the participants. It correctly identified $75 \%$ of the SUD patients (the rest were identified as cigarette smokers), $52.6 \%$ of the cigarette smokers (15\% of the smokers were identified as SUD patients and $32.4 \%$ as controls), and $83.3 \%$ of the controls (the rest were identified as cigarette smokers). On the whole, the correct identification of this analysis was highly significant $(71.4 \%$ represents an improvement of $38.1 \%$ over the chance level of $33.3 \%$; significance of Critical Ratio is 4.266, $\mathrm{p}<.001$ ).

This analysis identified two functions of which one was significant. This function included the COAT-Q beliefs about the self and about norms and rules (Eigenvalue 1.925), and accounted for $94.1 \%$ of the variance. The group centroids of this function (where a centroid represents the mean standardized score of the two COAT-Q-beliefs that entered the discriminant function) were 1.471 for the SUD patients, .200 for the cigarette smokers, and -1.384 for the controls, suggesting that this function differentiated mainly between SUD and control women.

A stepwise discriminant analysis with the COAT-Q-themes as predictors classified correctly $88.9 \%$ of the participants. It correctly identified $95 \%$ of the SUD patients (the rest were identified as cigarette smokers), $78.9 \%$ of the cigarette smokers (the rest were identified as controls) and $91.7 \%$ of the controls (the rest were identified as smokers). On the whole, the correct identification of this analysis was highly significant $(88.9 \%$ represents an improvement of $55.6 \%$ over the chance level of 33.3\%; significance of Critical Ratio is 6.16, $\mathrm{p}<.001$ ).

This analysis identified two significant functions (Eigenvalues 3.368 and .829 , respectively), accounting for $80.3 \%$ and $19.7 \%$ of the variance, respectively. The group centroids of the first function were 2.624 for the SUD patients, -1.123 for the cigarette smokers and -1.298 for the controls, and of the second function -.052 for the SUD patients, 1.232 for the cigarette smokers, and -.932 for the controls. Accordingly, the first function differentiated mainly the SUD patients from the controls, whereas the second function differentiated mainly between the cigarette smokers and the controls. 
Most COAT-Q-beliefs and theme-clusters were found to be significantly correlated with the EPQ-R-AS (results not shown).

\section{Discussion}

The present study shows significant differences between the two groups with addictive behaviors and the control group in all COAT-Q 25 themes and the four belief types. Specifically, compared with healthy controls, SUD patients show more COAT-Q-beliefs and themes identified as orienting towards addictiveness, in addition to elevated levels of anxiety, depression, and addictiveness (see Table 1). The high correlations found between the COAT-Q beliefs and themes and addictiveness (EPQ-R-AS) lend support to the potential of the COAT-Q to identify cognitions orienting towards addictiveness.

The COAT-Q clusters in the present study provide important insights into the inner world of SUD patients which turns out to be significantly different from that of healthy controls. The relevant contents form three clusters. The first represents an inclination of a vulnerable, confused and fearful individual to avoid emotions and needs that are conceived by her as highly negative, and to maintain distance from others. The second and third clusters seem to support in their contents the directionality of the first, in that they deal mainly with issues related to unworthiness and negative self-perception (cluster two), and to the inability to accept loving feelings from others, despite wishing to be able to do so (cluster three) .

The COAT-Q-themes underlying drug use in our sample are, thus, related primarily to the avoidance of negative emotionality and negative self-perception. This avoidance bears considerable resemblance to the mechanism of relief-craving [23], which postulates that individuals characterized by a high stress reactivity personality style who use substances, do so primarily to reduce anxiety and tension. Other studies have also found that drugs are often used as a "self-medication" mechanism, to increase the inclination to detach and move away from painful negative affects such as anxiety, depression, or aggressiveness $[4,10]$. The avoidance of negative emotionality in our patients may correspond also to Clonninger's [24] harm avoidance temperament that represents an inclination to avoid changes and novel conditions and to react mainly to stimuli evoking negative emotionality. Indeed, elevated harm avoidance has been found elevated in SUD patients in comparison to controls [9].

Our definition of chronic cigarette smoking complies with the accepted definition of the disorder, requiring daily smoking for at least several years [19]. The cigarette smokers in our study scored in-between the SUD patients and controls in two COAT-Q belief types (COAT-QBS, COAT-Q-N) and all three COAT-Q clusters (Table 1). This suggests that the underlying cognitive-motivational dispositions orienting towards chronic smoking have a lot in common with those relevant to addiction in general. Indeed, other studies have also demonstrated that tobacco users fare in-between heroin users and normal controls in adaptive and maladaptive personality attributes [25].

Further support for the validity of the COAT-Q in identifying addictive potential in cigarette smoking comes from two findings. Firstly, the cigarette smokers have differed from the controls in all but one COAT-Q-belief types (CAT-Q-G). Secondly, the use of stepwise discriminant analyses with the COAT-Q-beliefs and themes as predictors has not discriminated SUD patients from cigarette smokers.

Still, in keeping with previous studies [25], we found that for most variables introduced, cigarette smokers have not been not as severely impaired as the SUD patients. In particular, although cigarette smokers have been similar to the SUD patients with respect to their anxiety and depression levels at assessment, their overall inclination to display these traits was much lower.

The present study has been carried in women as it is a continuation of our study about addictiveness in EDs [18]. Considerable differences are found between male and female substance abusers [26]. Women typically begin using substances later than men, are strongly influenced by others to use, and enter treatment earlier than men [27]. Importantly, women have a significantly higher prevalence of comorbid psychiatric disorders such as depression, anxiety, and sexual abuse associated PTSD [28]. These disorders typically predate the onset of substance-abuse. For addicted women, who in addition suffer from greater stress reactivity [28], substances may be used to a greater extent to self-medicate negative emotions in comparison to men [27]. The avoidance and detachment of SUD women in our study from bear great resemblance with these findings. They lend further support for the relevance of relief-craving in addicted women, and suggest that the personality patterns of SUD women belong to an avoidant inhibited rather than a dysregulated sensation-seeking cluster.

The findings of our study should be interpreted with caution because of the relatively small number of participants, the inclusion of only females, the cross-sectional design, and the heterogeneity of the chronic smokers group (nicotine addiction was not an inclusion/ exclusion criterion). This could account for the relatively low potential of the COAT-Q-belief types, (although not the COAT-Q themes), to identify the chronic smokers from non-smoking controls.. In addition, the COAT-Q in the present study was constructed from another COquestionnaire, originally created for ED patients

Still, our study is a hypothesis generating research that has the advantage of investigating motivational dispositions underlying overt behaviors. Other advantages relate to the rigorous exclusion and exclusion criteria employed, and to the fact that the SUD patients were not under the influence of the drug when assessed, nor were they experiencing withdrawal or other acute physical and psychiatric symptoms.

In conclusion, we found significant differences in anxiety, depression, and addictiveness between SUD patients, chronic cigarette smokers, and controls. SUD patients were also highly different from controls in COAT-Q-beliefs and themes, with the cigarette smokers faring in most variables in-between the two other groups. These findings suggest that specific motivational dispositions can potentially direct towards addiction-related behaviors in SUDs, and to lesser extent also in chronic smoking. These motivations orient primarily toward avoidance and dissociation from a host of negative emotions and self-perceptions and from close contacts with others.

Future research should include larger samples of SUD individuals of both genders, and use a prospective and longitudinal design to assess the potential of the cognitive orientation model to identify not only motives underlying addiction, but also motives contributing to the ability to quit smoking and substance use.

\section{Funding}

The study was supported by a grant from the Israel Anti-Drug Authority.

\section{References}

1. Greydanus DE, Patel DE (2005) The adolescent and substance abuse: current concepts Dis Mon 392-431. [Crossref] 
2. Sher KJ, Bartholow BD, Wood MD (2000) Personality and substance use disorders: a prospective study. J Consult Clin Psychol 68: 818-829. [Crossref]

3. Gossop MR, Eysenck SB (1980) A further investigation into the personality of drug addicts in treatment. Br J Addict 75: 305-311. [Crossref]

4. Hatsukami D, Owen P, Pyle R, Mitchell J (1982) Similarities and differences on the MMPI between women with bulimia and women with alcohol or drug abuse problems. Addict Behav 7: 435-439. [Crossref]

5. Kranitz L (1972) Alcoholics, heroin addicts and nonaddicts; comparisons on the MacAndrew Alcoholism Scale of the MMPI. Q J Stud Alcohol 33: 807-809. [Crossref]

6. American Psychiatric Association (1994) Diagnostic and statistical classification of mental disorders: DSM-IV. Washington, DC: American Psychiatric Association.

7. Haller DL, Miles DR (2004) Personality disturbances in drug-dependent women: relationship to childhood abuse. Am J Drug Alcohol Abuse 30, 269-286. [Crossref]

8. Galen LW, Henderson MJ, Whitman RD (1997) The utility of novelty seeking, harm avoidance, and expectancy in the prediction of drinking. Addict Behav 22: 93-106. [Crossref]

9. Howard MO, Kivlahan D, Walker RD (1997) Cloninger's tridimensional theory of personality and psychopathology: applications to substance use disorders. J Stud Alcohol 58: 48-66. [Crossref]

10. Speranza M, Corcos M, Stephan P, Loas G, Perez-Diaz F, et al. (2004) Alexithymia, depressive experiences, and dependency in addictive disorders. Subst Use Misuse 39: 551-579. [Crossref]

11. Kreitler S, Shahar A, Kreitler H (1976) Cognitive orientation, type of smoker and behavior therapy of smoking. Br J Med Psychol 49: 167-175. [Crossref]

12. Kreitler H, Kreitler S (1982) The theory of cognitive orientation: Widening the scope of behavior prediction. In B. Maher \& W. GB. Maher (Eds.), Prog Exp Pers Res 11: 101-169. [Crossref]

13. Kreitler S, Schwartz R, Kreitler H (1987) The cognitive orientation of expressive communicability in schizophrenics and normals. J Commun Disord 20: 73-91. [Crossref]

14. Kreitler S, Bachar E, Canetti L, Berry E, Bonne O (2003) The cognitive-orientation theory of anorexia nervosa. J Clin Psychol 59: 651-671. [Crossref]
15. Kreitler S (2004) The cognitive guidance of behavior. In J. T. Jost, M. R. Banaji, \& D. A. Prentice (Eds.), Perspectivism in social psychology: The Yin and Yang of scientific progress (pp. 113-126). Washington, DC: American Psychological Association.

16. Kreitler S, Kreitler H (1994) Motivational and cognitive determinants of exploration. In H. Keller, K. Schneider \& B. Henderson (Eds.), Curiosity and exploration 259-284.

17. Davis C, Claridge G (1998) The eating disorders as addiction: a psychobiological perspective. Addict Behav 23: 463-475. [Crossref]

18. Ram A, Stein D, Sofer S, Kreitler S (2008) The cognitive orientation profile in bulimia nervosa and substance use disorders: similarities and differences. Eating Disorders: The Journal of Treatment and Prevention 16: 1-17.

19. Terracciano A, Costa PT Jr (2004) Smoking and the Five-Factor Model of personality. Addiction 99: 472-481. [Crossref]

20. Keuthen NJ, Niaura RS, Borrelli B, Goldstein M, DePue J, et al. (2000) Comorbidity, smoking behavior and treatment outcome. Psychother Psychosom 69: 244-250. [Crossref]

21. First MB, Spitzer RL, Gibbon M, Williams JBW (1995) Structured clinical interview for AXIS-1 DSM-IV disorders. New York State Psychiatric Institute, NY: Biometric Research Department.

22. Spielberger CD (1995) Revised State-Trait Personality Inventory (Revised STPI). Palo Alto, CA: Mind Garden.

23. Verheul R, van den Brink W, Geerlings P (1999) A three-pathway psychobiological model of craving for alcohol. Alcohol Alcohol 34: 197-222. [Crossref]

24. Cloninger CR (1987) A systematic method for clinical description and classification of personality variants. Arch Gen Psychiatry 44: 573-588. [Crossref]

25. Terracciano A, Löckenhoff CE, Crum RM, Bienvenu OJ, Costa PT Jr (2008) FiveFactor Model personality profiles of drug users. BMC Psychiatry 8: 22. [Crossref]

26. Tuchman E (2010) Women and addiction: the importance of gender issues in substance abuse research. J Addict Dis 29: 127-138. [Crossref]

27. Brady KT, Randall CL (1999) Gender differences in substance use disorders. Psychiat Clin North Am 22: 241-252. [Crossref]

28. Greenfield SF, Back SE, Lawson K, Brady KT (2010) Substance abuse in women. Psychiatr Clin North Am 33: 339-355. [Crossref]

Copyright: (C2017 Stein D. This is an open-access article distributed under the terms of the Creative Commons Attribution License, which permits unrestricted use, distribution, and reproduction in any medium, provided the original author and source are credited. 